\title{
A rational method for determining intermittency in the transitional boundary layer
}

\author{
Dhamotharan Veerasamy ${ }^{1} \cdot$ Chris Atkin $^{1}$
}

Received: 14 June 2019 / Revised: 25 September 2019 / Accepted: 20 November 2019 / Published online: 3 December 2019

(c) The Author(s) 2019

\begin{abstract}
A new rational procedure is proposed for determining the intermittency in the streamwise direction. One of the key parameters for the intermittency determination is the selection of a threshold value, which often involves a certain level of subjectivity. Here, a reliable way of choosing the threshold value in a more objective manner is proposed. The proposed approach involves a single threshold value, equal to the magnitude of the maximum laminar perturbation in the transitional flow. The results obtained are validated with the widely used dual-slope method. In this paper, the measurements are carried out on an experimental arrangement, involving the interaction of an upstream aerofoil wake with a downstream flat plate boundary layer. As a by-product of the study, a scaling parameter has been identified which captures the length of the transition zone as the proximity of the aerofoil in the wall-normal direction is varied.
\end{abstract}

\section{Graphic abstract}

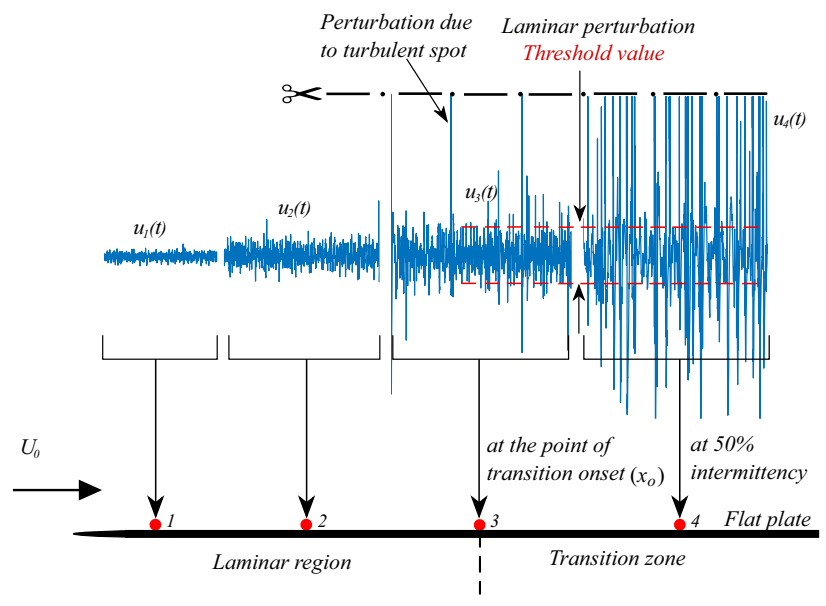

\section{Introduction}

In the study of turbulence, the intermittency factor $(\gamma)$ at a given point in the flow field is defined as the fraction of time the flow remains turbulent. The value of the intermittency factor varies from 0 to 1 , where a zero value represents a fully laminar region and the value of one represents a fully turbulent regime. The initial idea of intermittency

Dhamotharan Veerasamy

dhamotharan.veerasamy.1@ city.ac.uk

1 Department of Mechanical Engineering and Aeronautics, City, University of London, London EC1V 0HB, UK was proposed by Corrsin (1943) to differentiate the turbulent and non-turbulent patterns in axisymmetric turbulent jets. The first intermittency measurements were made by Townnsend (1948) in a turbulent wake. Though the idea of intermittency is derived from fully turbulent flows, its use became prominent in transitional boundary layer research since the discovery of turbulent spots in the transition zone by Emmons (1951). In the case of transition modelling, the intermittency distribution plays an essential role in capturing the length of the transition zone; however, the actual measurement of intermittency remains a challenge. Numerous techniques have been developed by many researchers (Hedley and Keffer 1974; Falco and Gendrich 1990; Kuan 
and Wang 1990; Schneider 1995; Zhang et al. 1996; Canepa et al. 2002; Fransson et al. 2005; Imayama et al. 2012) for the measurement of $\gamma$; but the methods proposed in the literature do not seem to work for all type of flows, resulting in no universal procedure for determining $\gamma$.

In the early days of transition research, the procedure followed for intermittency measurement was quite complex (Schubauer and Klebanoff 1956). It involved photographing the hot-wire signal from the oscilloscope screen and identifying the number and duration of bursts (occurrence of abrupt high-frequency peaks in the signal, considered to be the result of turbulent spots) in a given period of time. Subsequently, the intermittency was obtained by taking the ratio of the aggregate duration of the bursts to the total time of the acquired signal. Later, the intermittency was deduced using computer-based methods. Hedley and Keffer (1974) conceived a generic procedure for measuring the intermittency in a fully turbulent boundary layer, which is still used as a reference in transitional boundary layer research by many investigators. In their method, intermittency is determined by following a procedure consisting of four sequential steps that involve the selection and application of a detector function, a criterion function, a threshold value and an indicator function. These functions and the associated steps are explained as follows:

1. The detector function helps to discriminate between laminar and turbulent portions in the signal: this is called "sensitising" the signal. The time derivative of the velocity signals is commonly used as a detector function.

2. In the second step, the criterion function is obtained by smoothing the sensitised signal over a predefined time interval (usually in the order of 3-10 sampling intervals); this is done to avoid turbulent dropouts and spurious signals (laminar spikes) being taken into account during the analysis.

3. In the next step, a threshold value $\left(T^{h}\right)$ is chosen and compared with the criterion function; if the value of the criterion function exceeds the value of $T^{h}$, then that reading would be considered as turbulent.

4. Finally, the indicator function, $I(t)$, is obtained by assigning a value of 1 for this turbulent condition and zero for the remaining part of the signal. By averaging the indicator function over a given period of time, the intermittency is calculated.

This is the general procedure followed in many algorithms developed by several researchers for determining the intermittency using the time history signals obtained from hotwires/hot-films. Despite there being many algorithms for intermittency calculation, none of the methods is found to be robust. This is because of the subjectivities arising from the choice of detector function, the time interval in the criterion function and arbitrariness in the selection of the threshold value (Canepa et al. 2002). Among these, it is found that the selection of the threshold value, $T^{h}$ possesses the highest level of subjectivity as it strongly influences the intermittency determination. Some of the well-known intermittency calculation methods in transitional research are the TERA, MTERA, dual-slope and Probability Density Function (PDF) methods. In the TERA (Falco and Gendrich 1990) and MTERA (Zhang et al. 1995) methods, $T^{h}$ is determined by an arbitrary percentage of the root mean square (rms) value of the criterion function. Due to the arbitrariness in choosing the threshold percentage, this method has not been well received by other investigators. Kuan and Wang (1990) proposed the dual-slope method; this method involves a graphical search to identify the $T^{h}$ value. In this method, if the cumulative frequency distribution of the sensitised transitional signal is plotted against the detector function, then the distribution displays two different slopes and the point where the two different slopes intersect is considered to be the threshold. There are mixed reviews available for this method. Some researchers (Ramesh et al. 1996; Jahanmiri et al. 1991) succeeded in using this method, while others (Canepa et al. 2002; Fransson et al. 2005) have faced difficulty in identifying the two different slopes. Also, because of the graphical approach involved in this method, there is a certain level of subjectivity that exists in selecting the threshold value. However, at present, this method is widely used for various transitional flows. Next, in the PDF method (Solomon 1996), the $T^{h}$ value is selected from the intersection of two conditional PDFs obtained from the selected laminar and turbulent portions of the detector function measured at a given point. This method is easy to employ on the signals acquired from surface hot-films, since the surface films are free from the contamination of edge intermittency (Narasimha 1985) and their sensitivity is generally higher than that of hot-wires. Conversely, in this method, an initial, arbitrary threshold value has to be assumed for segregating the laminar and turbulent portion of the signal. Also, this method would not produce good results near the onset and breakdown of transition due to the absence of an intersection between the conditional PDFs of laminar and turbulent signal (Canepa et al. 2002). In another PDF method, the threshold value is chosen corresponding to the valley between the laminar and turbulent peaks observed in the PDF of the entire signal (Schneider 1995). This method tends to fail especially near the transition onset region where the existence of the valley becomes ambiguous. Further suggestions (Canepa et al. 2002) have been made to overcome the difficulties encountered in the above method, however, such modifications result in a time-intensive process to determine the intermittency. In the case of direct numerical simulations, Nolan and Zaki (2013) and Marxen and Zaki (2019) 
have employed an image analysis technique (so called Ostu's method, Otsu 1979) to arriving at a threshold value.

From the literature, it is clear that the proposed methods include a significant level of subjectivity in deciding the threshold value; also, the threshold values have to be re-defined for each station to determine the intermittency distribution. Further, the threshold values are not easily reproduced by other investigators using the same data or with the same experimental setup. Motivated by these problems, a new method is proposed in the present work using a more physical approach to determine the intermittency in the transitional zone. The advantages of the proposed approach when compared with those reported in the literature are as follows: (1) the method is simple, rational and the choice of threshold value is automatic, (2) a single threshold value can be used for the whole transition region, thus reducing analysis time, and (3) threshold values and intermittency distributions should be quantitatively reproducible by other investigators with the same data or under similar experimental conditions.

The proposed methodology is evaluated on an experimental arrangement involving the interaction between an aerofoil wake and a downstream flat plate boundary layer. Here, the aerofoil wake acts as a source of free-stream disturbance, which triggers laminar-turbulent transition on the flat plate boundary layer. This type of transition is generally referred as wake-induced transition, commonly seen in multi-element aerofoils (during aircraft take-off and landing phases) and turbomachinery flows. The rig allows the interaction to be controlled by simply changing the proximity of the aerofoil to the flat plate, which in turn presents a range of test cases to check the robustness of the proposed method. To this end, the streamwise intermittency distribution in the downstream, transitional boundary layer is studied in detail by applying the proposed approach to hot-wire measurements. Furthermore, the consistency of the new technique reveals that some interesting scaling characteristics between the proximity of the aerofoil and transition region are reported. It is worth to mention at this point, to the best of our knowledge, this kind of experimental setup, particularly focusing on transition research, was not previously reported in the literature.

\section{Experimental setup}

All the experiments reported in this paper were performed in the low-turbulence wind tunnel at City, University of London. This is a closed-circuit wind tunnel with test section dimensions $0.924 \times 0.915 \times 3.66 \mathrm{~m}$. The section width increases from 0.924 to $0.940 \mathrm{~m}$, over the length of $3.66 \mathrm{~m}$ in the downstream direction. The operating velocity inside the test section can vary from 0.5 to $25 \mathrm{~m} / \mathrm{s}$; for the present experiment, a free-stream velocity $\left(U_{0}\right)$ of $20 \mathrm{~m} / \mathrm{s}$ was considered.

An aluminium flat plate with dimensions $2082 \times 915 \times 10$ $\mathrm{mm}$ was used in the present experiment. The flat plate was mounted vertically in the test section and it was fitted with an elliptical leading edge of about $63 \mathrm{~mm}$ in length, which was made up of an asymmetric, wooden biconvex surface. To maintain the stagnation point on the measurement side of the leading edge and to maintain a zero pressure gradient, a trailing-edge flap of $110 \mathrm{~mm}$ length was employed. Eventually, overall length of the flat plate $(l)$ considered for the present experiment was, $2082+63+110=2255 \mathrm{~mm}$.

Ahead of and above the leading edge of the flat plate, a NACA 0014 aerofoil, with a chord length $(c)$ of $250 \mathrm{~mm}$, was placed at zero degree angle of attack, shown schematically in Fig. 1. The vertical separation between the aerofoil and the flat plate is denoted as the 'height' $\left(h_{w}\right)$ and the horizontal separation is denoted as the 'overlap' $\left(x_{w}\right)$. The streamwise and wall-normal measurement stations are defined using a coordinate system, $x-y$, having its origin at the leading edge of the flat plate. The aerofoil spanned the entire test section, both ends meeting the tunnel walls. Furthermore, the aerofoil was tripped at around $25 \%$ of the chord using a sandpaper roughness strip (average roughness

Fig. 1 Schematic representation of the experimental setup (plan view)

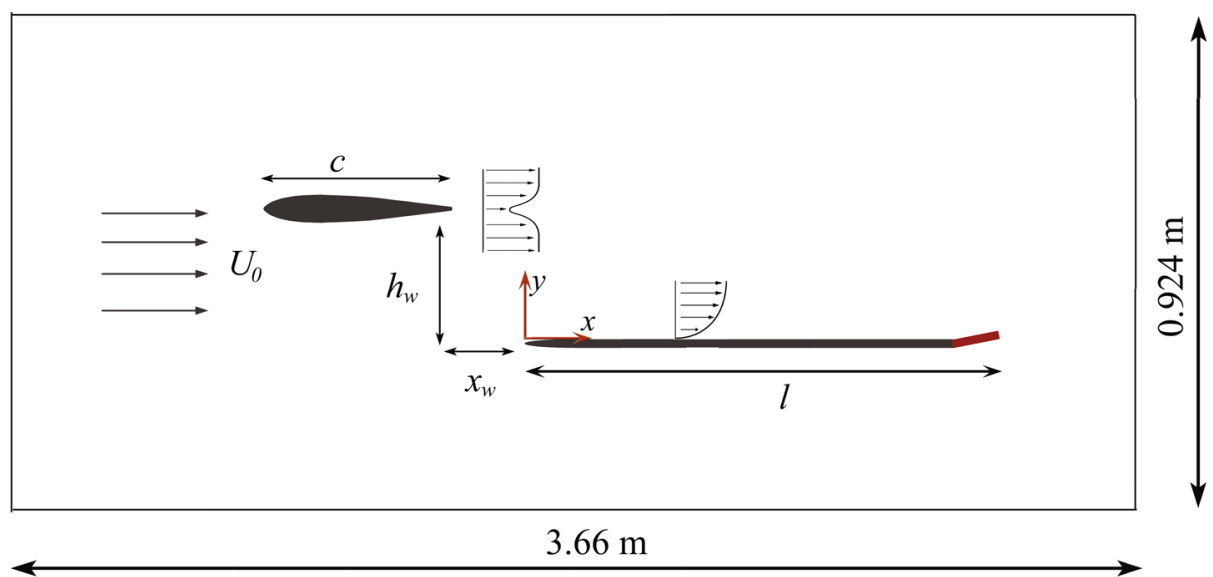


height $=425 \mu \mathrm{m})$ to avoid vortex shedding. With the flat plate and aerofoil mounted in the tunnel, the free-stream turbulence intensity in the streamwise direction, at $20 \mathrm{~m} / \mathrm{s}$, was measured as $0.015 \%$ (in the frequency range of $5 \mathrm{~Hz}-5$ $\mathrm{kHz}$ ). This compares with $0.007 \%$ when the tunnel is empty.

All velocity measurements were carried out using a Dantec DISA 55M01, Constant Temperature Anemometry (CTA) unit. The hot-wire probe (boundary layer type) featured a single tungsten wire (diameter $5 \mu \mathrm{m}$, length 1.25 $\mathrm{mm}$ ). The probe was calibrated in the wind tunnel using the velocity obtained from the pitot-static tube. During the calibration process, the upstream aerofoil was removed to avoid wake interference. The raw signals acquired from the probe were sampled at a frequency of $10 \mathrm{kHz}$ and passed through an analogue filter (Krohn-Hite, model 3360 series), where the signal was band-pass filtered between $5 \mathrm{~Hz}$ and $5 \mathrm{kHz}$. The lower bound of $5 \mathrm{~Hz}$ was chosen to remove the DC component and electronic noise; while the upper bound of $5 \mathrm{kHz}$ was chosen to avoid aliasing. In all measurements, the signal was acquired for a total duration of $30 \mathrm{~s}$ to obtain converged steady state. A National Instruments DAQ card was used to digitise the acquired signal and a user interface was developed in LabVIEW for controlling the wind tunnel speed and the traverse mechanism for the boundary layer probe. The laboratory was air-conditioned but the temperature inside the (closed-circuit) wind tunnel increased by up to $3{ }^{\circ} \mathrm{C}$ when compared with the ambient temperature at which the calibration was conducted. This was compensated for by performing a temperature correction on the voltage signal. For the present experiments, the individual uncertainties in calibration, linearization (curve fit) and analogue to digital conversion were found to be $0.06,0.0095$ and 0.0035 $\mathrm{m} / \mathrm{s}$ respectively. The combined standard uncertainty for the hot-wire velocity was, thus, calculated as $0.3 \%$ of freestream speed. To traverse the probe inside the test section, a 3 -axis system was used with independent movement in the streamwise $(x)$, wall-normal $(y)$ and spanwise $(z)$ directions. The smallest step size in the streamwise and spanwise directions was $10 \mu \mathrm{m}$, while that in the wall-normal direction was $1.25 \mu \mathrm{m}$. A laser positioning system was employed to avoid accidental contact between the probe and the surface of the flat plate during near-wall boundary layer measurements.

\subsection{Aerofoil wake and flat plate pressure gradient}

The purpose of the aerofoil in the present experiment is to generate the wake as a source of free-stream disturbance. Therefore, to elucidate the characteristics of the aerofoil wake, measurements were first carried out in the wake region without the flat plate. Figure 2 shows the mean velocity profiles of the wake measured at various streamwise distances downstream of the trailing edge. All the stations are normalised by chord length (for this particular measurement, origin of the co-ordinate system $(x, y)$ is taken as the aerofoil trailing edge). This measurement was obtained at a free-stream velocity of $20 \mathrm{~m} / \mathrm{s}$; the corresponding Reynolds number based on the aerofoil chord $\left(R e_{c}\right)$ is $3.4 \times 10^{5}$. In the figure, $U_{0}, \bar{U}$ and $u_{o}\left(=U_{0}-\bar{U}\right)_{\max }$ represent the freestream velocity, time-averaged mean velocity and maximum defect velocity respectively. $y_{0.5}$ is the wall-normal location corresponding to the defect velocity being equal to $0.5 u_{o}$. For comparison, the self-similar solution of the wake mean velocity obtained by Wygnanski et al. (1986) is also plotted in the figure. It can be observed that the present results are in excellent agreement with the plane wake measurements of Wygnanski.

To ascertain the nature of the wake turbulence, the power spectral density was obtained. Figure 3 shows the spectral distribution at $x / c=0.8$ for various wall-normal stations. Near the wake core region $(\eta=0.1)$, the spectrum displays the characteristics of isotropic turbulence, where most of the energy is associated with low to mid-range frequencies. Around and away from the edge of the wake $(\eta>2)$, a bandwidth of mid-range frequencies (recognised as a slightly spread-out bump) becomes dominant in the spectrum, but varies with $\eta$. Therefore, depending upon the proximity of the aerofoil to the flat plate, the nature of the disturbance inducing transition on the plate will vary. In the present experiments, it should be noted that the aerofoil is positioned in such a way that the wake core does not interact directly with the leading edge of the flat plate in order to avoid the flow becoming turbulent right from the leading edge. For the minimum aerofoil height $\left(h_{w}=20 \mathrm{~mm}\right)$ considered in the present experiment, the leading edge of the flat plate lies at a normalised distance $\eta=3.2$ from the wake centreline.

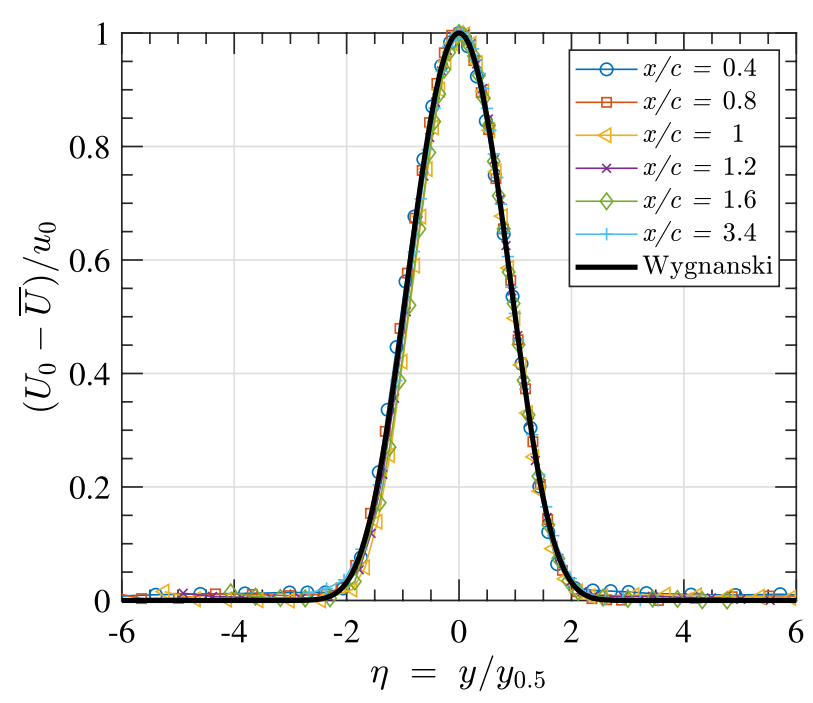

Fig. 2 Self-similar mean velocity profiles of the aerofoil wake compared with Wygnanski et al. (1986) 


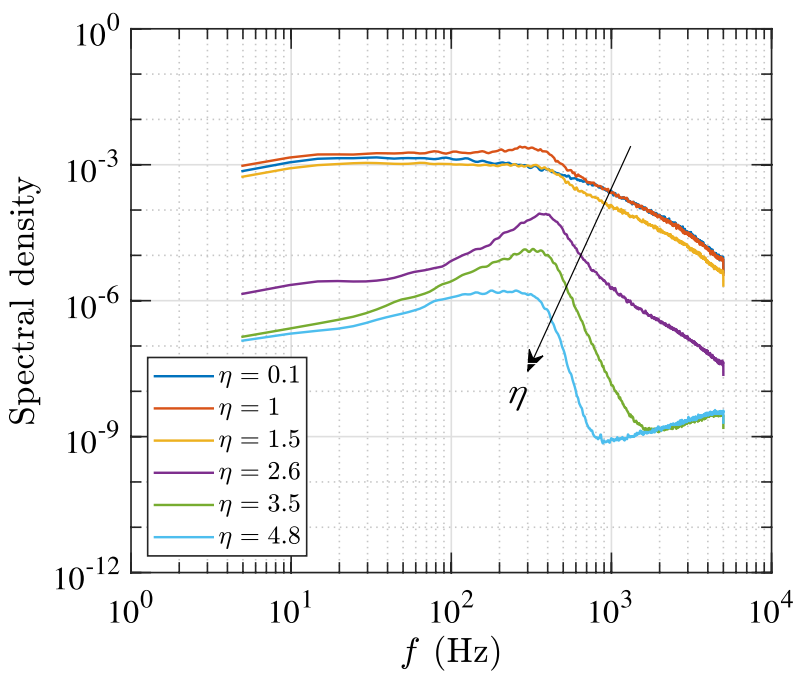

Fig. 3 Power spectral density of the aerofoil wake measured at $x / c=0.8$ for various wall-normal stations; $R e_{c}=3.4 \times 10^{5}$

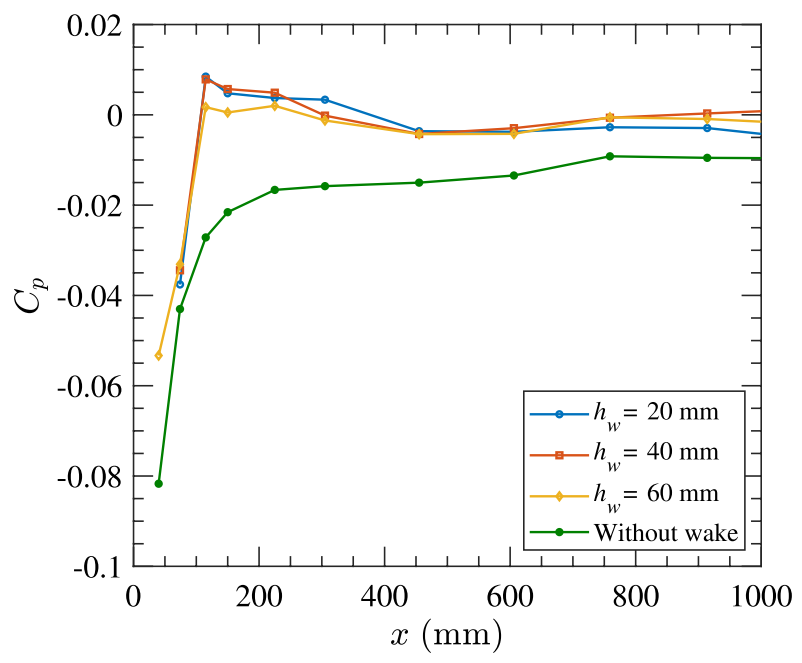

Fig. 4 Surface pressure distribution on the flat plate for various $h_{w}$ of the aerofoil

Introducing the aerofoil upstream of the flat plate may disturb the surface pressure distribution on the plate. The $C_{\mathrm{p}}$ (coefficient of pressure) distribution on the flat plate, obtained for various aerofoil heights at $R e_{c}=3.4 \times 10^{5}$, is shown in Fig. 4. It is evident that the presence of the aerofoil slightly altered the pressure distribution over the leading edge; however, it was confirmed using tuft flow visualisation that it did not lead to flow separation. Also, downstream of the leading edge, a significant region of laminar flow was observed on the flat plate for all the heights $\left(h_{w}=20,25,30\right.$, 35 and $40 \mathrm{~mm}$ ) considered in the experiment. For the present study, measurements were obtained between $x=100 \mathrm{~mm}$ and $x=900 \mathrm{~mm}$, since the pressure distribution remained approximately constant in this region. Further, the overlap $\left(x_{w}\right)$ between the aerofoil and flat plate was maintained at $25 \%$ of the chord for all the experiments.

\section{Rational method for streamwise intermittency determination}

As highlighted in Sect. 1, the selection of threshold value $\left(T^{h}\right)$ plays a vital role in intermittency determination and it has a direct effect on the intermittency distribution. For instance, selection of a lower $T^{h}$ will indicate greater intermittency in the laminar regime and earlier transition onset in the flow. On the other hand, the selection of a higher $T^{h}$ might indicate that a fully turbulent flow regime is still transitional. Therefore, it is crucial to have an optimum $T^{h}$ to accurately determine the intermittency. As discussed in Sect. 1, most of the intermittency determination methods in the literature involve an arbitrary constant or a subjective approach to determine the threshold value.

To overcome the problem with subjective selection of the threshold value, an objective approach is introduced in this paper. The basic idea behind this approach is illustrated in Fig. 5 using the streamwise fluctuating velocity signals obtained at four different points on the flat plate, designated as 1,2, 3 and 4 , whose fluctuating velocity signals are shown by $u_{1}(t), u_{2}(t), u_{3}(t)$ and $u_{4}(t)$, respectively. Points 1 and 2 are chosen in the upstream region where the flow tends to be laminar, and the points 3 and 4 are taken from the downstream region of the plate, generally falling in the transition region. It is known that, as we move downstream in the boundary layer, the magnitude of the fluctuations in the flow also increases. This is clearly demonstrated in Fig. 5, especially from points 1 to 3 , where the magnitude of the fluctuation velocity increases in the downstream distance, until we reach point 3 and observe turbulent spots (spiky signals). By observing the signals at the transition onset (point 3 ) and the middle of the transition zone (point 4), it is easy to distinguish the perturbations due to turbulent spots from the laminar perturbations. An interesting point is that the laminar perturbations (within the red dashed line) do not seem to increase in magnitude between the transition onset point (point 3) and the middle of the transitional zone (point 4), in contrast to the increasing amplitude of these perturbations in the laminar region (points 1\&2). Such a characteristic was observed in all of the experiments conducted in this work. This observation prompts an assumption that the magnitude of the laminar perturbations remains constant throughout the transition region. In this regard, it is proposed to choose the magnitude of the laminar perturbation at the transition onset point as a threshold value for intermittency determination. Such a threshold value would retain only the turbulent fluctuations from the transitional signals, eliminating the 


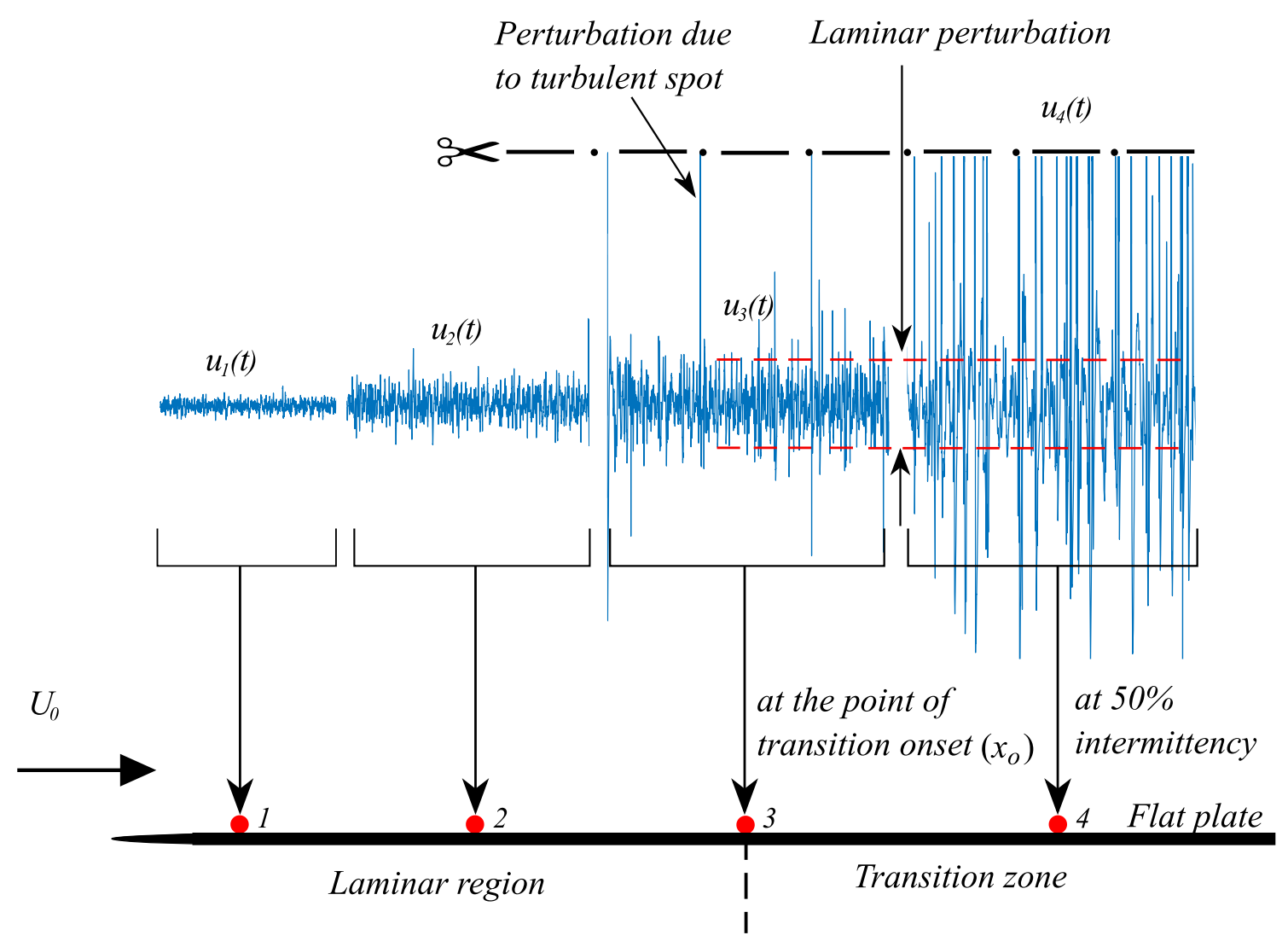

Fig. 5 The proposed approach to choosing the threshold value for intermittency determination. All the fluctuation signals are plotted to the same scale and here obtained for the case $h_{w}=35 \mathrm{~mm}$. The

laminar perturbations. This approach has been applied to the present measurements to determine the intermittency. It will be demonstrated later in this paper that the obtained results look sensible and that the derived intermittency matches well with a detailed visual analysis of the fluctuating signal.

\subsection{Transition onset and breakdown point}

To apply the proposed approach, as a first step, it is essential to identify a method for determining the transition onset point $\left(x_{o}\right)$ and from there extract the magnitude of the laminar perturbation signal. In hot-wire measurements, transition onset is usually determined visually by observing the near-wall fluctuating signals and identifying the streamwise location where the first turbulent spot (spiky signal, similar to Fig. 5) occurs. This method would be time consuming and prone to human error (i.e., failing to notice the spot). To overcome this issue, in the present experiment, a method based on streamwise skewness distribution is used to define transition onset.

Streamwise skewness distribution has been previously employed to determine the transition zone in the experiments fluctuation due to turbulent spots (spiky signal) in $u_{3}(t)$ and $u_{4}(t)$ are cropped at the higher end, to accommodate the figure within the page

on multi-element aerofoils (Bertelrud and Anders 2002) and turbine blades (Halstead et al. 1997; Gomes et al. 2015). However, the definition of the skewness parameter is different in each paper. In the present experiment, following Gomes et al. (2015), skewness is defined as the ratio of the third moment to the cube of the root mean square of the fluctuating velocity, given by $\left(\overline{u^{3}}\right) / u_{\mathrm{rms}}^{3}$. Using the signals obtained from surface hot-films, Gomes et al. showed that the locations of maximum (positive) and minimum (negative) skewness correspond to transition onset $\left(x_{o}\right)$ and breakdown points $\left(x_{b}\right)$, respectively. Though hot-wire measurements are predominantly used in the current work, it is expected that the skewness-based method described above would also work in the present scenario, since Matsubara et al. (1998) showed that the intermittency distribution in the wall-normal direction remains constant up to $y / \delta^{*}=1\left(\delta^{*}\right.$ is the displacement thickness). To substantiate this idea, the skewness distribution obtained using hot-wire signals at $y / \delta^{*}=0.5$ for the case $h_{w}=25 \mathrm{~mm}$ is plotted in Fig. 6 , and the corresponding time history of the fluctuating velocity signals is given in Fig. 7.

From Fig. 6, the location of maximum skewness occurs at $x / l=0.11$. At the same time, from Fig. 7, downstream of 


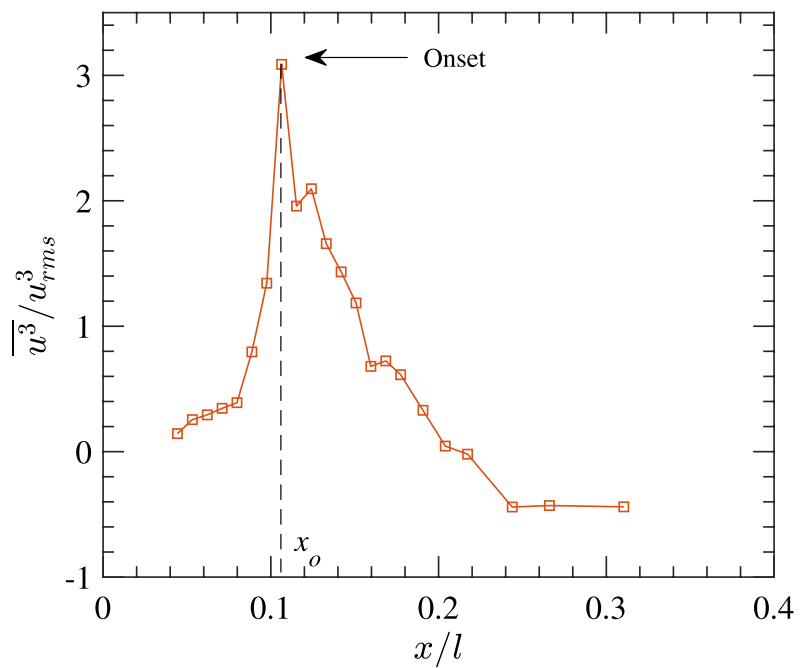

Fig. 6 Streamwise skewness distribution, measured at $y / \delta^{*}=0.5$, for the case $h_{w}=25 \mathrm{~mm}, x_{w}=0.25 c$ and $R e_{c}=3.4 \times 10^{5}$. Here, ' $l$ ' is the overall length of the flat plate

$x / l=0.11$, the occurrence of abrupt spikes in the fluctuation signals (indication of turbulent spots) is more frequent, while upstream of $x / l=0.11$, these spiky signals are very rare. For the other aerofoil heights $\left(h_{w}\right)$ considered in the experiment, a similar kind of behaviour was noticed. This confirms that, in the present experiment, the location of maximum skewness corresponds with the transition onset point $\left(x_{o}\right)$.

On the other hand, from Fig. 7, breakdown (fully turbulent) seems to occur at $x / l>0.24$ where the corresponding skewness value (Fig. 6) is negative, remaining constant further downstream. This observation is contrary to the observations of Gomes et al., where they noted, at the point of breakdown, a negative peak in the skewness distribution. This difference in behaviour could be attributed to the fact that, in the present experiment, measurements are made on a zero pressure gradient boundary layer; whereas, Gomes et al. investigated a typical turbine blade pressure distribution. Furthermore, Österlund (1999) reported that, for a zero pressure gradient turbulent boundary layer, the skewness obtained at the outer region (above the logarithmic layer) remained constant in the streamwise direction. Conversely, for varying adverse pressure gradient, Harun (2012) found that the skewness did not remain constant in the downstream direction. The literature indicates that the skewness distribution in the turbulent regime of the present experiment follows Österlund (1999) and the differences with Gomes et al. may be due to the difference in the streamwise pressure gradient. From these arguments, the point at which the skewness reaches a constant level is considered as the breakdown point in the present experiment. In the case of $h_{w}=25 \mathrm{~mm}$, the skewness distribution in Fig. 6 shows that the breakdown

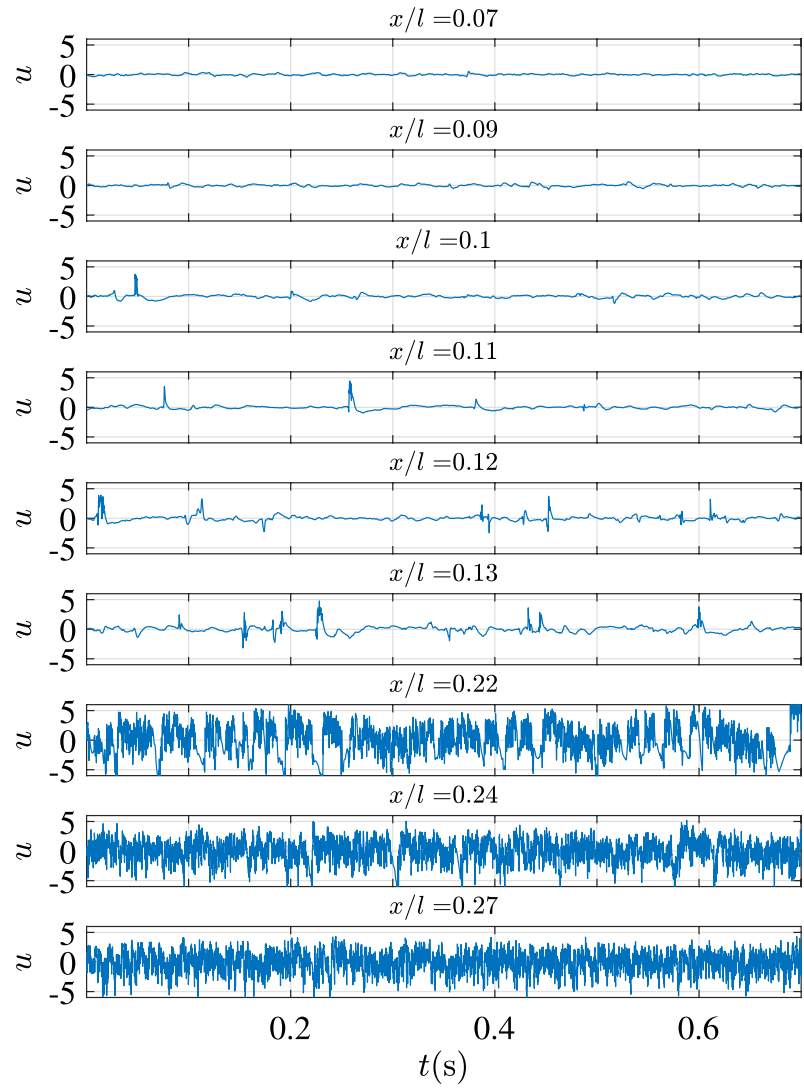

Fig. 7 Streamwise velocity fluctuations during laminar to turbulent transition. Geometry conditions corresponding to Fig. 6. The actual signal is acquired for $30 \mathrm{~s}$, but for the sake of brevity, it is shown only for $0.7 \mathrm{~s}$

point occurs at $x / l>0.24$, which can be verified visually from the corresponding fluctuation signals shown in Fig. 7.

The skewness distributions for different aerofoil heights are shown in Fig. 8. Figure 9 shows the transition onset and breakdown points, obtained from the location of maximum skewness and from the beginning of negative plateau region, respectively, for different $h_{w}$. It can be observed that a linear dependence exists between the onset and breakdown points and $h_{w}$, but with different slopes. Thus the length of the transition region appears to be increasing with increasing $h_{w}$. These results will be cross-checked with the intermittency results in later sections.

Uncertainty in Fig. 9: since the streamwise measurement stations are discrete (step size of $50-100 \mathrm{~mm}$ ) it is difficult to determine the exact skewness peak $\left(x_{o}\right)$ or the point at which the skewness becomes plateau $\left(x_{b}\right)$. This in turn leads to uncertainty in determining the transition onset and breakdown points. In Fig. 9; these uncertainties are quantified (error bars) using the streamwise step size used in the experiment. 


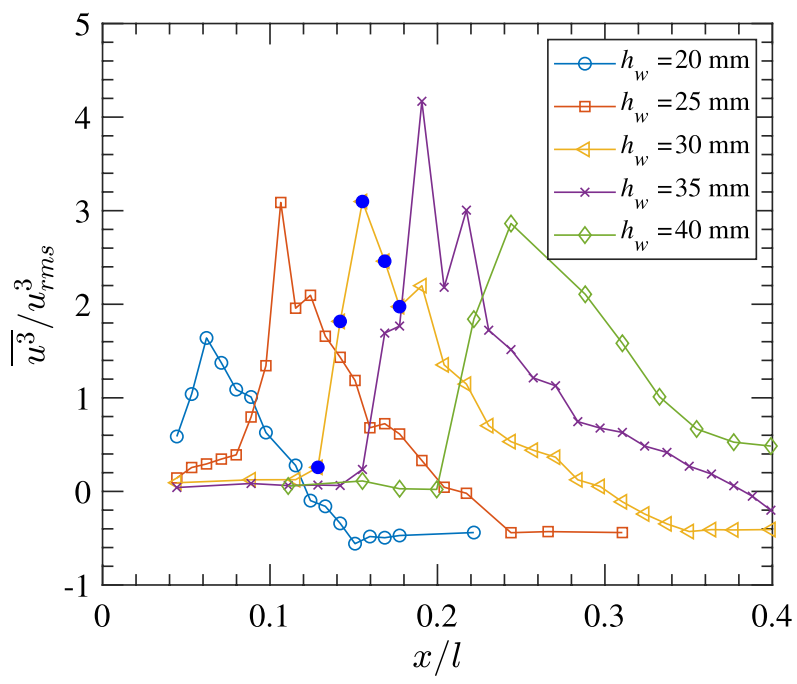

Fig. 8 Skewness distribution for various aerofoil heights; $x_{w}=0.25 c$, $R e_{c}=3.4 \times 10^{5}$ and $y / \delta^{*}=0.5$. The streamwise location of the skewness peak is considered to be the transition onset point $\left(x_{o}\right)$. The location where the skewness reaches a constant level is taken as the breakdown point $\left(x_{b}\right)$. The solid blue marker is explained in Sect. 3.2

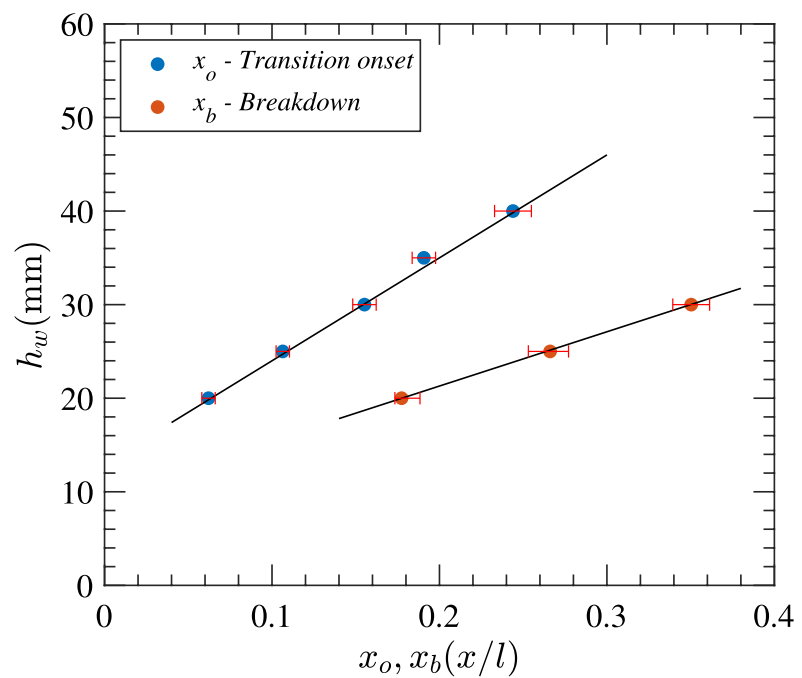

Fig. 9 Variation of transition onset $\left(x_{o}\right)$ and breakdown $\left(x_{b}\right)$ locations with respect to the height $\left(h_{w}\right)$ between the aerofoil and flat plate; $x_{w}=0.25 c, \operatorname{Re}_{\mathrm{c}}=3.4 \times 10^{5}$ and $y / \delta^{*}=0.5$

\subsection{Threshold value $\left(T^{h}\right)$ and intermittency $(\gamma)$}

It was discussed in Sect. 1 that the available intermittency determination methods require different $T^{h}$ values for each station in the streamwise direction. For the method proposed here, it is emphasised that a single threshold value is sufficient for determining the streamwise development of intermittency, namely the magnitude of laminar perturbation measured at the transition onset point $\left(x_{o}\right)$. Firstly, to demonstrate the validity of the choice of threshold value, the streamwise intermittency distributions are obtained for the case $h_{w}=30 \mathrm{~mm}$ using five different $T^{h}$ values, $T_{0}^{h}, T_{-1}^{h}, T_{-2}^{h}$, $T_{+1}^{h}, T_{+2}^{h}$ chosen at locations corresponding to the transition onset point $\left(x_{o}\right)$, two stations upstream of the onset point $\left(x_{o-1}\right.$ and $\left.x_{o-2}\right)$, and two stations downstream of the onset $\left(x_{o+1}\right.$ and $\left.x_{o+2}\right)$. The $x / l$ values of these stations are 0.16 , $0.14,0.13,0.17$ and 0.18 , respectively, and the corresponding skewness values are marked in blue symbols in Fig. 8 . Finally, the resulting streamwise intermittency distributions based on these five different threshold values are compared with the dual-slope method (Kuan and Wang 1990) for validation purpose. As a side note, all the $T^{h}$ values and the intermittency distributions are obtained at the same wallnormal location, $y / \delta^{*}=0.5$.

\subsection{1 $T^{h}$ from onset point, $x_{o}\left(T_{0}^{h}\right)$}

The raw fluctuating velocity signals, $u(t)$, acquired at the transition onset point $\left(x_{o}\right)$ contains both laminar perturbations and occasional turbulent spots, as shown in Fig. 10a. According to the proposed method, the laminar perturbation alone is considered for determining the $T^{h}$ value. Therefore, to discard the perturbations due to turbulent spots, the signals are first sensitised by double differentiating with respect to time and then squaring, $\left.\left(\mathrm{d}^{2} u / \mathrm{d} t^{2}\right)^{2}\right|_{x_{o}}=D(t)_{x_{o}}$ Consequently, only the high-frequency signals, typically due to turbulent spots, are amplified, which can then be clearly distinguished from the laminar perturbations, as observed in Fig. 10b. The time interval of these high-frequency signals $\left(\Delta t_{a}\right)$ can be determined using the condition $D(t)_{x_{o}}>2 D_{x_{o, \mathrm{rms}}}$, where $D_{x_{o, \mathrm{rms}}}$
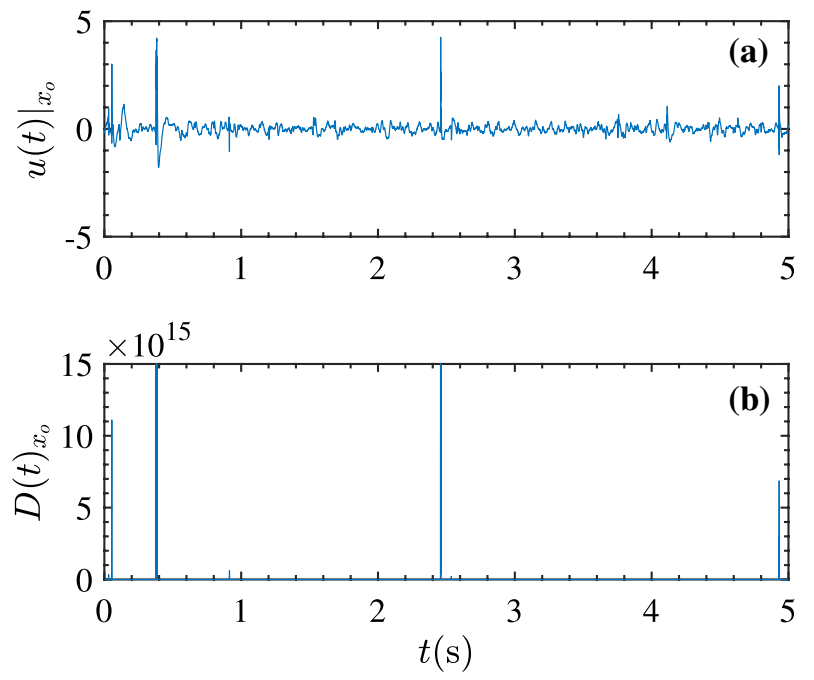

Fig. 10 a Raw fluctuation velocity obtained at $x_{o} \quad(x / l=0.16$, $y / \delta^{*}=0.5$ ) for the case $h_{w}=30 \mathrm{~mm}$. b Corresponding second derivative, $D(t)_{x_{o}}=\left.\left(\mathrm{d}^{2} u / \mathrm{d} t^{2}\right)^{2}\right|_{x_{o}}$ of the fluctuating velocity in a 
is the rms value of $D(t)_{x_{o}}$. Furthermore, to include the turbulent dropouts (hollow red markers in Fig. 11) and the trails of the high-frequency signals (solid red markers in Fig. 11), a window time of 3 sample points each to the left and right of the signal is added with $\Delta t_{a}$. The choice of this specific window time is based on Kolmogorov time scale used for obtaining the criterion function, also discussed in the later part of this section. Eventually, by discarding the signals in $\Delta t_{a}$ from $D(t)_{x_{o}}$, laminar perturbation, $D(t)_{x_{o}, L}$ alone is extracted. The threshold value, $T_{0}^{h}$, is then obtained by taking the rms of $D(t)_{x_{o}, L}$, denoted as $D_{x_{o}, L, \mathrm{rms}}$.

\subsection{2 $T^{h}$ from the stations downstream of onset, $x_{o+1}$ and $x_{o+2}\left(T_{+1}^{h}\right.$ and $\left.T_{+2}^{h}\right)$ :}

For the stations downstream of the onset point $\left(x_{o+1}\right.$ and $\left.x_{o+2}\right)$, a similar procedure to that described above can be adopted to eliminate the turbulent spots and extract the laminar perturbations alone. This would in turn yield the corresponding threshold values, $T_{+1}^{h}$ and $T_{+2}^{h}$ given by $D_{x_{o+1}, L, \mathrm{rms}}$ and $D_{x_{o+2}, L, \text { rms. }}$

\subsection{3 $T^{h}$ from the stations upstream of onset, $x_{o-1}$ and $x_{o-2}$ $\left(T_{-1}^{h}\right.$ and $\left.T_{-2}^{h}\right)$}

Upstream of the onset location, turbulent spots are not likely to appear in the fluctuating velocity signal; hence, the rms value of the sensitised raw fluctuating velocity signal can be directly considered as a $T^{h}$ value. Therefore, $T_{-1}^{h}$ and $T_{-2}^{h}$ values, chosen from one station upstream of the onset, $\left(x_{o-1}\right)$, and two stations upstream of the onset, $\left(x_{o-2}\right)$, are determined from $D_{x_{o}-1, \mathrm{rms}}$, and $D_{x_{o}-2, \text { rms }}$, respectively.

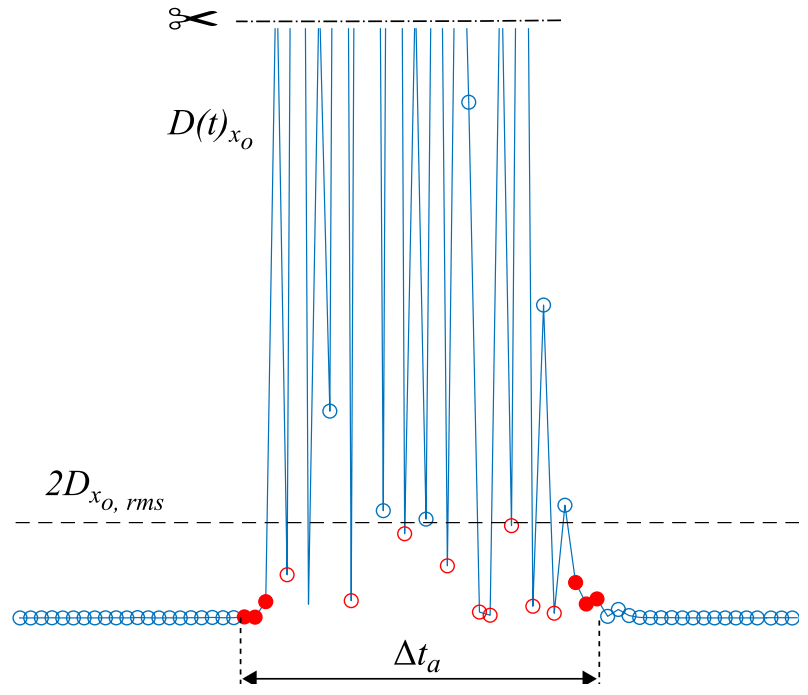

Fig. 11 Time interval of the high-frequency signal including the turbulent dropouts (hollow red markers) and trailing signal (solid marker)
Once the $T^{h}$ value is obtained, then the generic procedure conceived by Hedley and Keffer (1974) for determining the intermittency is followed. Firstly, the fluctuating velocity signals obtained at $y / \delta^{*}=0.5$ are sensitised using $D(t)_{x}=\left.\left(\mathrm{d}^{2} u / \mathrm{d} t^{2}\right)^{2}\right|_{x}$. Then, the sensitised signal is smoothed (moving average) within the sampling time interval of $7 \Delta t=0.0007 \mathrm{~s})$, which here is approximately 230 times the Kolmogorov time scale, $T_{k}$. (By following Turns et al. 1996, $T_{k}$ is defined in Eq. (1), where $T_{I}$ refers to the integral time scale, which was obtained using the autocorrelation of the fluctuating velocity signals in the fully turbulent regime.) Thereafter, the chosen threshold value is applied to the smoothed signal, which in turn yield the indicator function, $I(t)$ given in Eqs. (2) and (3). Eventually, by integrating the indicator function (Eq. 4) for the whole signal, the intermittency $\gamma$ is determined.

$\frac{T_{k}}{T_{I}} \sim R e_{t}^{-3 / 4}$,

where $R e_{t}=u_{\mathrm{rms}} \times\left(U_{0} T_{I} / v\right)$

$I(t)=\left\{\begin{array}{l}0, D(t)_{x} \leq T^{h} \\ 1, D(t)_{x}>T^{h}\end{array}\right.$

$\gamma=\frac{1}{T} \int_{0}^{T} I(t) \mathrm{d} t$

The resulting streamwise intermittency distributions for the five $T^{h}$ values, $T_{-2}^{h}, T_{-1}^{h}, T_{0}^{h}, T_{+1}^{h}$ and $T_{+2}^{h}$ are referred as $\gamma_{-2}, \gamma_{-1}, \gamma_{0}, \gamma_{+1}$ and $\gamma_{+2}$ respectively and are plotted in Fig. 12. It can be observed that the streamwise intermittency distribution corresponding to the threshold value chosen at onset point, $\gamma_{0}$, matches very well with that of the dual-slope method. Further, the intermittency distributions corresponding to the downstream threshold values $\gamma_{+1}$ and $\gamma_{+2}$ are also found to agree reasonably well with that of the dual-slope values. From this observation, it can be argued that, if the extracted laminar perturbations are used to determine the threshold value, one can obtain accurate values of intermittency distribution $\gamma(x)$. This, in turn, supports the assumption that the laminar perturbation remains constant in the transition zone. On the other hand, looking at the intermittency distributions $\gamma_{-2}$ and $\gamma_{-1}$, it can be seen that $\gamma_{-1}$ matches well, but $\gamma_{-2}$ does not. It could be reasoned that, when one goes upstream of the onset point, the magnitude of the laminar perturbations would be less than at the onset point, resulting in lower threshold values for farther upstream points. This is clear in the erroneous intermittency distribution, $\gamma_{-2}$, where the upstream laminar regions are shown as being intermittent. Ultimately, by observing all the intermittency distributions in Fig. 12, it can be concluded that a threshold value chosen at the transition onset point, or 


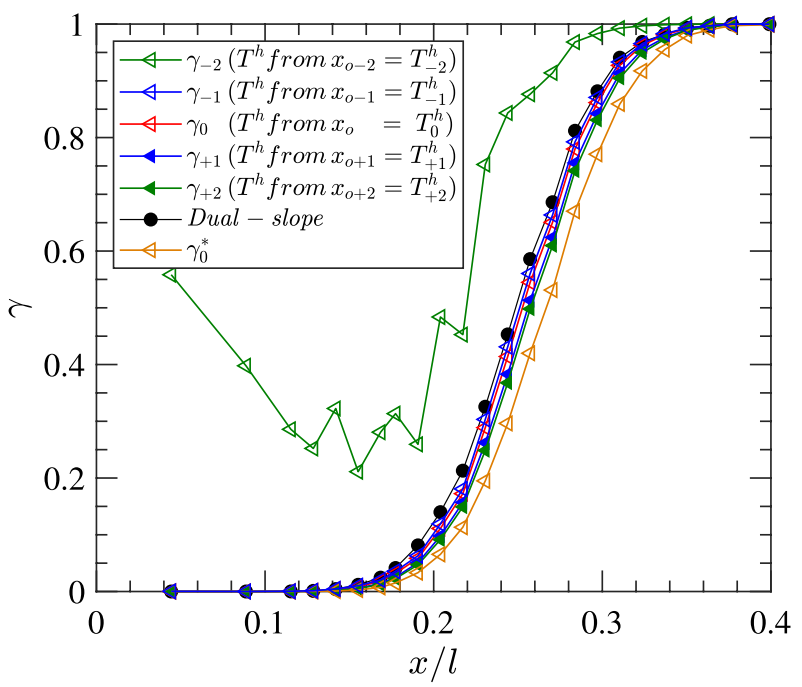

Fig. 12 Comparison of the streamwise intermittency distributions obtained using the proposed method and the dual-slope method; The geometric conditions are $y / \delta^{*}=0.5$ and $h_{w}=30 \mathrm{~mm}$

very close to the onset point, results in a repeatable intermittency distribution.

The reader may find the present approach somewhat subjective in two respects: first, distinguishing between the laminar and turbulent signals at, or downstream of, the onset point; second, the choice of sampling time interval for smoothing the sensitised signal (window time). In addressing the first concern, it is worth emphasising that the turbulent spots are few in number close to the onset point, thus alleviating the level of subjectivity involved. On the other hand, if the threshold value is chosen one station upstream of the onset, then there will be no spots, and the level of subjectivity is completely eliminated. Even if the threshold value is determined without eliminating those spots at the onset point $\left(T_{0}^{h}=D_{x_{0}, \text { rms }}\right)$, a reasonable trend in the intermittency distribution still emerges, as shown in Fig. 12, denoted as $\gamma_{0}^{*}$. In respect of sampling window size, the literature suggests a typical range between 3 and $10 \Delta t$. For example, Kuan and Wang (1990) and Keller and Wang (1995) applied $3 \Delta t$, Ramesh et al. (1996) used $5 \Delta t$ and both Zhang et al. (1996) and Canepa et al. (2002) used $10 \Delta t\left(250 T_{k}\right)$. The effect of window time on the calculated intermittency distribution is demonstrated in Fig. 13. It can be seen that, for the current experimental data, the choice of window time has a negligible effect on the determined intermittency distribution. Therefore, with this substantial evidence, we believe that the present method is rational and objective although its successful application to different data sets would obviously be required to give confidence for more general application.

Having verified the proposed approach, the intermittency distribution is obtained for different aerofoil heights $\left(h_{w}\right)$,

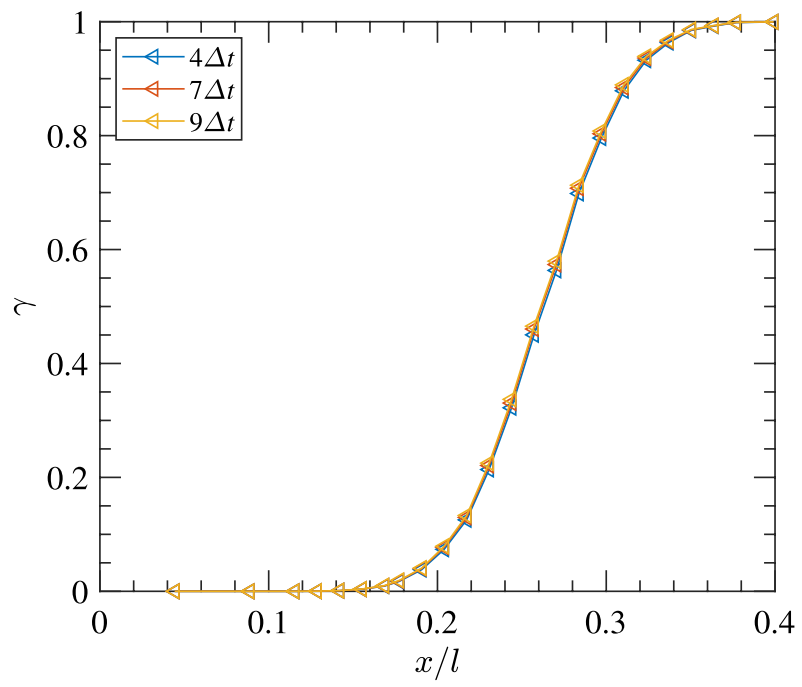

Fig. 13 The effect of window time on the streamwise intermittency distributions. The geometric conditions are $y / \delta^{*}=0.5$ and $h_{w}=30$ $\mathrm{mm}$

using the $T^{h}$ value obtained at corresponding onset points, shown in Fig. 14. It can be seen that the results obtained using the proposed method, for various $h_{w}$, match well with those of the dual-slope method; thus, it shows the confidence in the proposed idea. On the other hand, the present results do not follow the Narasimha (1957) hypothesis of concentrated breakdown, particularly at the beginning and end of the transition zone. This mismatch could be explained by differences in spot production rate. In Narasimha's theory,

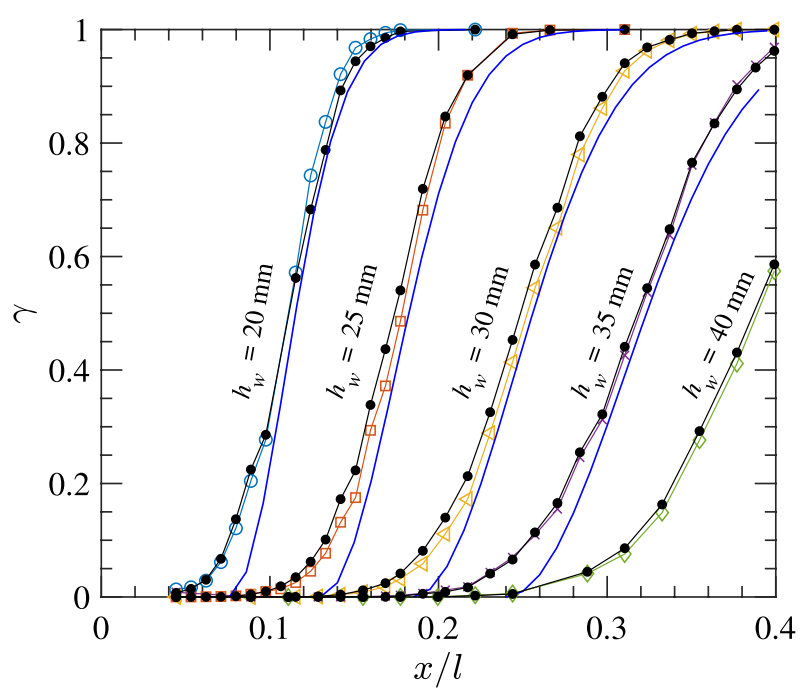

Fig. 14 Streamwise intermittency distributions for various heights $\left(h_{w}\right)$ of the aerofoil. Symbols: hollow markers-using proposed method; solid marker-dual-slope method ; solid line-Narasimha (1957) 
the intermittency distribution was modelled based on natural transition, where the forcing disturbance is only from free-stream turbulence. However, in the present experiment (wake-induced transition), in addition to the free-stream, the wake acts as another source of disturbance which may increase the spot production rate in the boundary layer. Consequently, higher intermittency is found in the present experiment when compared with Narasimha (1957).

From the present intermittency $(\gamma)$ distribution in Fig. 14, transition onset and breakdown points are defined as the stations where $\gamma$ corresponds to $1 \%$ and $99 \%$, respectively, and from there the length of the transition zone $\left(x_{l t}\right)$ can be calculated. In Fig. 15, $x_{l t}$ obtained from the present method and the skewness method (Fig. 9) are compared. The differences lie within experimental uncertainty although there is a systematic trend in the difference which would need a longer streamwise domain to address fully. However, we believe that these results support the approach of determining the transition region from the skewness distribution. Additionally, the method proposed for the threshold selection accurately captures the intermittent nature of the flow.

From Figs. 9 and 15, $x_{o}, x_{b}$ and $x_{l t}$ all vary linearly with respect to the height of the aerofoil above the plate. This suggests that some scaling exists between $h_{w}$ and the transition zone. To ascertain the scaling character, the obtained skewness and intermittency distributions are normalised with the transition onset point in Figs. 16 and 17. Interestingly, all the curves collapse onto a single curve, except for a slight deviation at $h_{w}=20 \mathrm{~mm}$. It can be reasoned that a decrease in $h_{w}$ may increase the turbulent intensity interacting with the boundary layer edge of the flat plate to a point at which a different scaling is required, as suggested by Fransson et al. (2005). In the present experiment, just

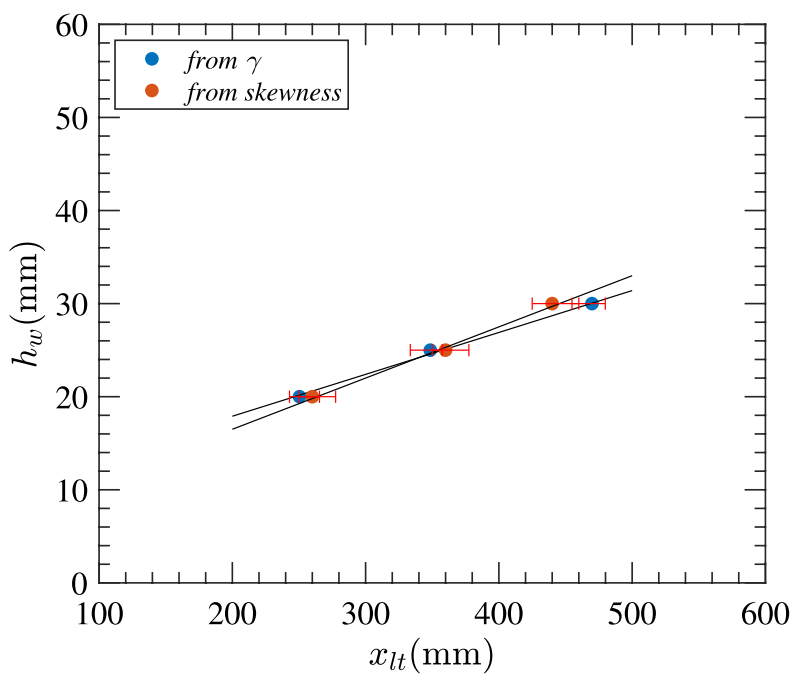

Fig. 15 Compared the length of the transition zone $\left(x_{l t}\right)$ obtained from Figs. 9 and 14

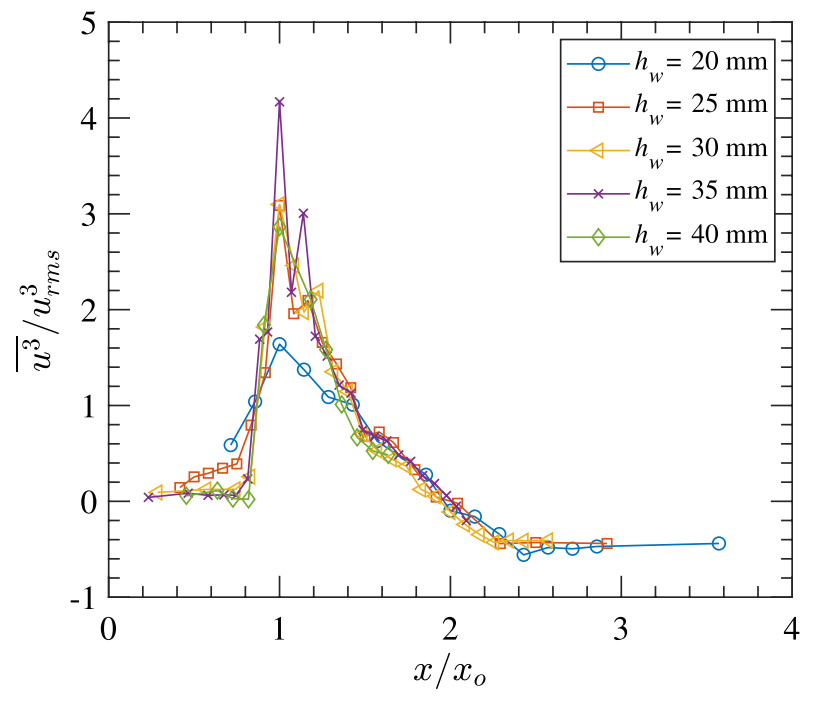

Fig. 16 Skewness distribution for various heights of the aerofoil scaled with transition onset point

before transition onset, the turbulent intensity at the edge of the boundary layer was found to be $2.2 \%$ for $h_{w}=20 \mathrm{~mm}$, $1.5 \%$ for $h_{w}=25 \mathrm{~mm}$ and less than $1 \%$ for other aerofoil heights. Fransson et al have shown that, for increasing freestream turbulence, the proper scaling for the intermittency distribution includes the length of the transition zone. By applying a revised scaling following their suggestion, the intermittency distribution at $h_{w}=20 \mathrm{~mm}$ can be made to collapse with the other cases, as seen in Fig. 18. Furthermore, the nature of transition at $h_{w}=20 \mathrm{~mm}$ was found to be of bypass type; while for the aerofoil height $h_{w}=40 \mathrm{~mm}$ and

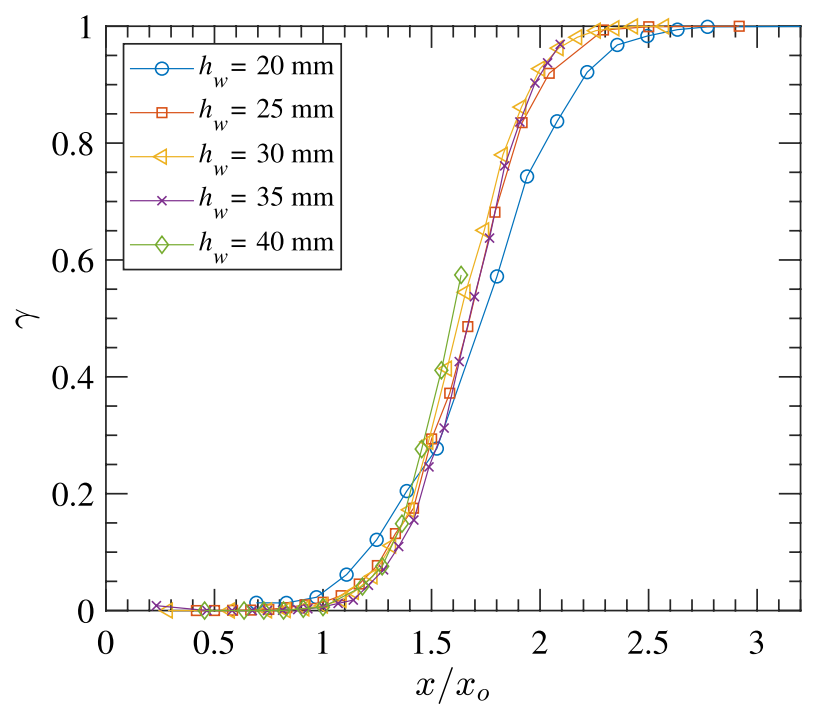

Fig. 17 Streamwise intermittency distribution for various heights of the aerofoil scaled with transition onset point 


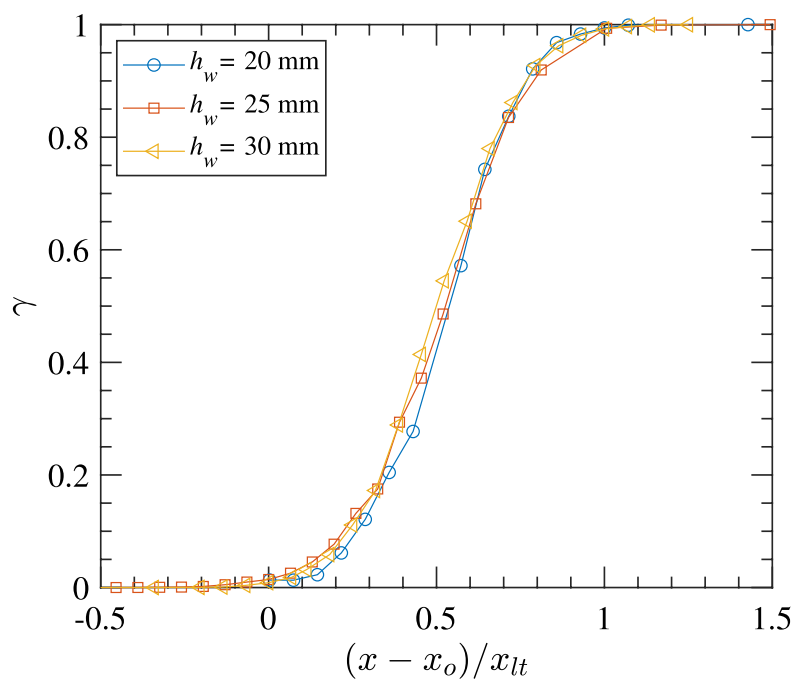

Fig. 18 Streamwise intermittency distribution based on the scaling given in Fransson et al. (2005)

beyond, we noticed mixed (natural and bypass) transitional characteristics-a paper on this topic is in preparation.

\section{Summary and conclusions}

Most of the intermittency estimation methods presented in the literature are subjective, with some level of arbitrariness in choosing the threshold value. In addition, those methods require a different threshold value to be chosen for each streamwise station, making the procedure laborious and time consuming. In the present work, a rational and objective technique is proposed to address the shortcomings involved in the other methods. The underlying idea is to detect the maximum laminar perturbation in the transitional flow and adopt this as the threshold for determining intermittency. It has been shown that using the threshold value obtained from the laminar perturbations at or around the onset point captures the transition zone very well and matches with the commonly used dual-slope method. These results in turn justify the assumption that the magnitude of the laminar perturbations remains constant in the transition zone, which might be an inherent physical characteristic of the transitional boundary layer.

The specific advantages of the proposed method when compared with the other existing methods are as follows: (1) a single threshold value is sufficient for the streamwise intermittency determination in the entire flow, thus reducing the analysis time when compared with other methods where a threshold value has to be chosen for each streamwise station. (2) The threshold value is selected in an objective way, which can be quantitatively reproducible by other investigators with the same data or under similar experimental conditions. In other methods, there is a certain level of subjectivity in determining the threshold value, for example graphical approaches (dual-slope method) or constants involved in the criterion function (TERA/MTERA), which make quantitative comparisons somewhat arbitrary. (3) This method is simple, rational, and the threshold value is effectively self-selecting. In other methods, as discussed in the introduction, there can be difficulty in determining the threshold value near the onset and breakdown points. The principal limitation of the present method is that the proposed approach cannot be used for determining intermittency distribution in the wall-normal direction, $\gamma(y)$.

Additionally, an interesting scaling characteristic of the transitional boundary layer is observed in the present experiment. The skewness and intermittency distributions obtained for various heights of the aerofoil scale well with the onset location. Further, it was found that the length of the transition zone also increases linearly with respect to the aerofoil height above the flat plate.

The robustness of the present intermittency determination method needs to be verified by application to other types of flows, which would also help to substantiate the assumptions involved in the present approach. On the other hand, it is clear from the results presented here that, in the case of wake-boundary layer interactions, the present approach works very well and should be valuable for transitional boundary layer research in turbomachinery and other multielement flows.

Acknowledgements We would like to acknowledge the financial support of Innovate UK under Grant ref. 113024, Enhanced Fidelity Transonic Wing, led by Airbus. We also thank Professor. Michael Gaster for his comments, suggestion and fruitful discussion during this work. Also the first author is grateful to Dr. Sathiskumar Anusuya Ponnusami for his linguistic help during the manuscript preparation.

Open Access This article is distributed under the terms of the Creative Commons Attribution 4.0 International License (http://creativeco mmons.org/licenses/by/4.0/), which permits unrestricted use, distribution, and reproduction in any medium, provided you give appropriate credit to the original author(s) and the source, provide a link to the Creative Commons license, and indicate if changes were made.

\section{References}

Bertelrud A, Anders J (2002) Transition documentation on a three-element high-lift configuration at high Reynolds numbers-analysis. NASA CR-2002-211438

Canepa E, Ubaldi M, Zunino P (2002) Experiences in the application of intermittency detection techniques to hot-film signals in transitional boundary layers. In: The 16th symposium on measuring techniques in transonic and supersonic flow in cascades and turbomachines', pp 2077-2085

Corrsin S (1943) Investigation of flow in an axially symmetric heated jet in air. NACA Wartime Reports, W-94 
Emmons H (1951) The laminar-turbulent transition in a boundary layer-part 1. J Aeronaut Sci 18(7):490-498

Falco R, Gendrich C (1990) The turbulence burst detection algorithm of Z. Zaric. Near-wall turbulence, pp 911-931

Fransson JH, Matsubara M, Alfredsson PH (2005) Transition induced by free-stream turbulence. J Fluid Mech 527:1-25

Gomes RA, Stotz S, Blaim F, Niehuis R (2015) Hot-film measurements on a low pressure turbine linear cascade with bypass transition. $\mathrm{J}$ Turbomach 137(9):091007

Halstead DE, Wisler DC, Okiishi TH, Walker GJ, Hodson HP, Shin H-W (1997) Boundary layer development in axial compressors and turbines: Part 1 of 4 composite picture. J Turbomach 119(1):114-127

Harun Z (2012) The structure of adverse and favourable pressure gradient turbulent boundary layers. $\mathrm{PhD}$ thesis. University of Melbourne, Australia

Hedley TB, Keffer JF (1974) Turbulent/non-turbulent decisions in an intermittent flow. J Fluid Mech 64(4):625-644

Imayama S, Alfredsson PH, Lingwood RJ (2012) A new way to describe the transition characteristics of a rotating-disk boundarylayer flow. Phys Fluids 24(3):031701

Jahanmiri M, Rudra Kumar S, Prabhu A (1991) A method for generating the turbulent intermittency function. Report 91 FM 13, Dept. of Aerospace Engineering, Indian Institute of Science, Bangalore

Keller FJ, Wang T (1995) Effects of criterion functions on intermittency in heated transitional boundary layers with and without streamwise acceleration. J Turbomach 117(1):154-165

Kuan C, Wang T (1990) Investigation of the intermittent behavior of transitional boundary layer using a conditional averaging technique. Exp Thermal Fluid Sci 3(2):157-173

Marxen O, Zaki TA (2019) Turbulence in intermittent transitional boundary layers and in turbulence spots. J Fluid Mech 860:350-383

Matsubara M, Alfredsson PH, Westin KJA (1998) Boundary layer transition at high levels of free stream turbulence. In: ASME 1998 international gas turbine and aeroengine congress and exhibition, pp V001T01A063-V001T01A063
Narasimha R (1957) On the distribution of intermittence in the transition region of a boundary layer. J Aeronaut Sci 24:711-712

Narasimha R (1985) The laminar-turbulent transition zone in the boundary layer. Prog Aerosp Sci 22(1):29-80

Nolan KP, Zaki TA (2013) Conditional sampling of transitional boundary layers in pressure gradients. J Fluid Mech 728:306-339

Österlund JM (1999) Experimental studies of zero pressure-gradient turbulent boundary layer flow. PhD thesis. KTH Royal Institute of Technology, Sweden

Otsu N (1979) A threshold selection method from gray-level histograms. IEEE Trans Syst Man Cybern 9(1):62-66

Ramesh O, Dey J, Prabhu A (1996) Transitional intermittency distribution in a three-dimensional constant pressure diverging flow. Exp Fluids 21(4):259-263

Schneider S (1995) Improved methods for measuring laminar-turbulent intermittency in boundary layers. Exp Fluids 18(5):370-375

Schubauer GB, Klebanoff PS (1956) Contributions on the mechanics of boundary-layer transition. NACA Technical Report, 1289

Solomon WJ (1996) Unsteady boundary layer transition on axial compressor blades. PhD thesis. University of Tasmania, Australia

Townnsend A (1948) Local isotropy in the turbulent wake of a cylinder. Aust J Chem 1(2):161-174

Turns SR et al (1996) An introduction to combustion, vol 499. McGraw-hill, New York

Wygnanski I, Champagne F, Marasli B (1986) On the large-scale structures in two-dimensional, small-deficit, turbulent wakes. J Fluid Mech 168:31-71

Zhang D, Chew Y, Winoto SH (1995) A proposed intermittency measurement method for transitional boundary layer flows. Exp Fluids 19(6):426-428

Zhang D, Chew Y, Winoto S (1996) Investigation of intermittency measurement methods for transitional boundary layer flows. Exp Thermal Fluid Sci 12(4):433-443

Publisher's Note Springer Nature remains neutral with regard to jurisdictional claims in published maps and institutional affiliations. 